\section{ALBI Score as a Novel Tool in Staging and Treatment Planning for Hepatocellular Carcinoma: Advantage of ALBI Grade for Universal Assessment of Hepatic Function}

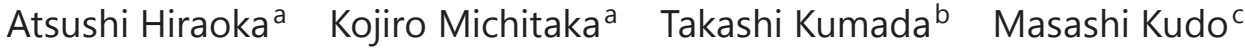 \\ ${ }^{a}$ Gastroenterology Center, Ehime Prefectural Central Hospital, Matsuyama, b Department of \\ Gastroenterology, Ogaki Municipal Hospital, Ogaki, and ' Department of Gastroenterology \\ and Hepatology, Kindai University Faculty of Medicine, Osaka, Japan
}

Thank you for your interest in our article titled "Albumin-bilirubin (ALBI) grade as part of the evidencebased clinical practice guideline for HCC of the Japan Society of Hepatology: a comparison with the liver damage and Child-Pugh classification" [1].

Huo et al. have sent an important message in their Letter to the Editor. Incorporation of models based on albumin-bilirubin (ALBI)/platelet-albumin-bilirubin (PALBI) grading, calculated using platelet count, serum albumin, and serum total bilirubin, into current HCC staging systems should be considered in order to refine their prognostic accuracy. There is no doubt that a new detailed assessment tool for hepatic function is needed in order to increase predictive accuracy for prognosis. We agree with their proposal.

Liu et al. [2] reported that the accuracy of PALBI grade was superior to that of ALBI. It is well known that the prognosis of a patient with HCC depends both on hepatic functional reserve and tumor factors. Thus, in order to address the issues raised by Huo et al., we used modified Japan integrated staging (JIS) scoring, which is calculated based on Child-Pugh classification and tumor node metastasis stage (TNM) of the Liver Cancer Study Group of Japan, 6th edition, as well as ALBI-TNM (ALBI-T) [3] and PALBI-TNM (PALBI-T) to validate the comparison with ALBI and PALBI, after excluding 1 patient whose platelet count was unknown $(n=3,494)$. Kaplan-Meyer curves for ALBI-T and PALBI-T are presented in Figure 1, and they show similar stratification abilities for prognosis. The c-index was the same value as shown in ALBI-T and PALBI-T scoring, while Akaike's information criterion (AIC) for ALBI-T was slightly better than that for PALBI-T (Table 1). When AIC and c-index were assessed for each etiology, PALBI-T was better in HCC patients with the hepatitis $\mathrm{C}$ virus (HCV), while ALBI-T was better in those with hepatitis B virus (HBV), as well as those both without HBV and HCV. Thus, the prognostic predictive values may vary slightly for each etiology. HCV and HBV was detected in 64.6 and $14.5 \%$, respectively, of the patients in our cohort, whereas HBV was the most common etiology in the report by Liu et al. [2].

Of note, it cannot be denied that ALBI grade calculated with only 2 factors, albumin and bilirubin, was not inferior to PALBI and presents a simpler tool for assessment of hepatic function. In fact, a good correlation between ALBI and PALBI scores was observed ( $r=0.820,95 \%$ CI: 0.809-0.831, $p<0.0001)$. Moreover, there

Atsushi Hiraoka, MD, PhD

Gastroenterology Center

Ehime Prefectural Central Hospital

83 Kasuga-cho, Matsuyama, Ehime 790-0024 (Japan)

E-Mail hirage@m.ehime-u.ac.jp 


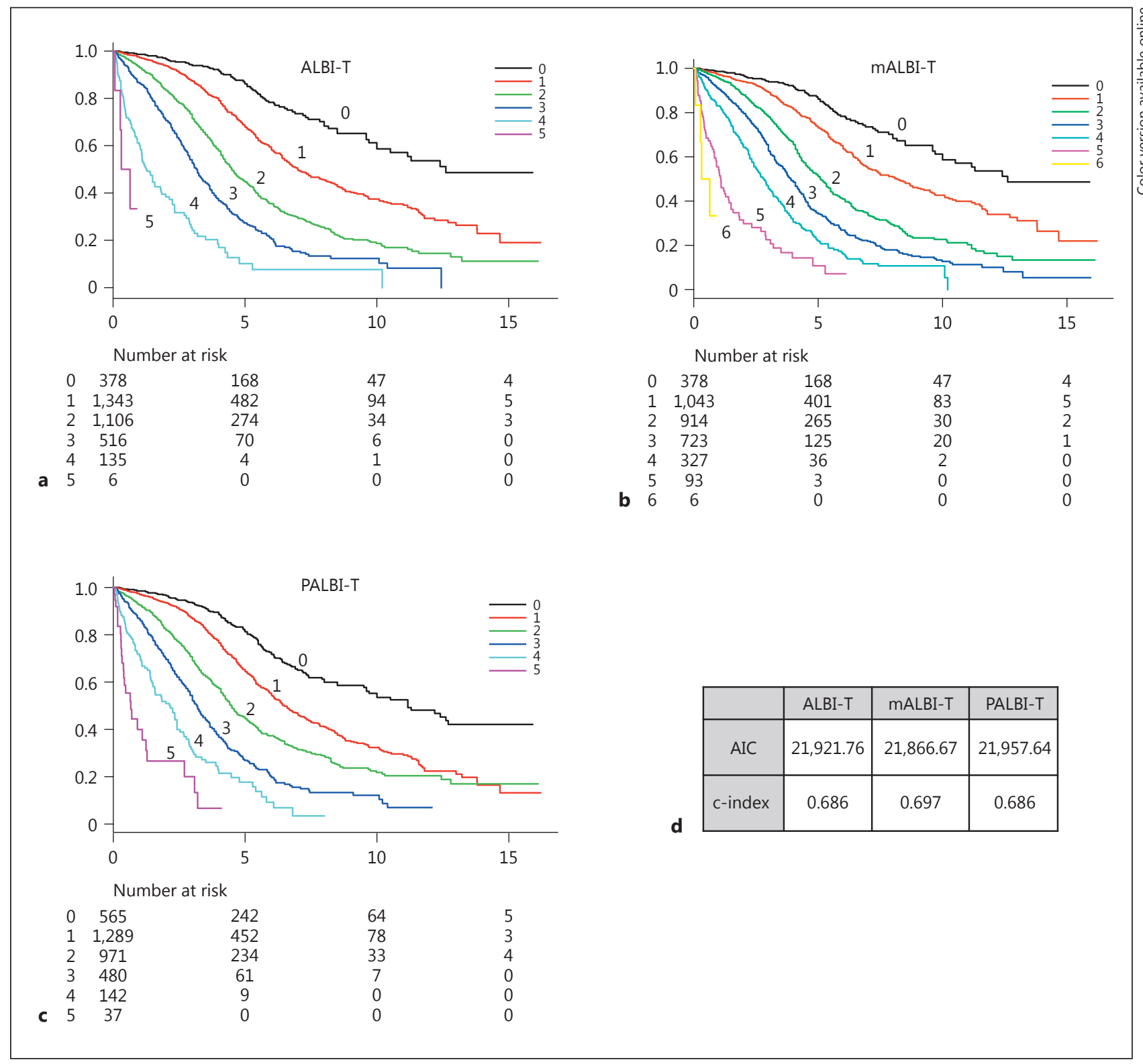

Fig. 1. Overall survival with each assessment tool in combination with tumor node metastasis classification (ALBI-T/mALBI-T/PALBI-T). Overall survival (OS) stratified by ALBI-T (a), mALBI-T (b), and PALBI-T (c) scores. With each, the stratification ability for prognosis was good. Akaike's information criterion (AIC) and c-index were better with mALBI-T compared to the others for assessment of prognosis of the entire patient cohort (d).

is an additional advantage with ALBI grade. A modified version has been proposed with 4 grades $(1,2 \mathrm{a}$, 2b, and 3) (modified ALBI [mALBI] grade) for more detailed assessment based on the cut-off value (ALBI score $=-2.270)$ for ICG-R15 $(<30 \%)$ [4]. In the results of additional analyses, AIC and c-index of mALBI/ mALBI-T were better than the others (Fig. 1; Table 1).

To improve the accuracy of the prognostic predictive value of HCC staging systems, further validation studies and investigations with international collaboration as well as larger patient cohorts are required. As noted by Huo et al., we agree that incorporation of ALBI/mALBI/PALBI-based models into current HCC staging systems is recommended in order to refine the prognostic accuracy of each system. 


\section{Liver Cancer}

Table 1. AIC and c-index with ALBI/mALBI/PALBI grades and ALBI-T/mALBI-T/PALBI-T scores

\begin{tabular}{l|l}
\hline \multicolumn{2}{l}{ Liver Cancer 2017;6:377-379 } \\
\hline DOI: 10.1159/000481212 & $\begin{array}{l}\text { @ 2017 S. Karger AG, Basel } \\
\text { www.karger.com/lic }\end{array}$ \\
\hline
\end{tabular}

Hiraoka et al.: ALBI Score as a Novel Tool in Staging and Treatment Planning for HCC: Advantage of ALBI Grade for Universal Assessment of Hepatic Function

\begin{tabular}{|c|c|c|c|}
\hline & ALBI & mALBI & PALBI \\
\hline \multicolumn{4}{|l|}{ TNM stage I } \\
\hline AIC & $3,641.5$ & $3,606.6$ & $3,663.7$ \\
\hline c-index & 0.638 & 0.680 & 0.635 \\
\hline \multicolumn{4}{|l|}{ TNM stage II } \\
\hline AIC & $9,342.6$ & $9,330.2$ & $9,375.8$ \\
\hline c-index & 0.613 & 0.630 & 0.613 \\
\hline \multicolumn{4}{|c|}{ TNM stage III } \\
\hline AIC & $4,606.31$ & $4,604.3$ & $4,609.1$ \\
\hline c-index & 0.577 & 0.592 & 0.585 \\
\hline \multicolumn{4}{|c|}{ TNM stage IV } \\
\hline AIC & 774.9 & 772.2 & 775.8 \\
\hline c-index & 0.597 & 0.633 & 0.621 \\
\hline \multicolumn{4}{|l|}{ Total } \\
\hline AIC & $22,222.2$ & $22,176.0$ & $22,240.6$ \\
\hline \multirow[t]{2}{*}{ c-index } & 0.609 & 0.630 & 0.619 \\
\hline & ALBI-T & mALBI-T & PALBI-T \\
\hline \multicolumn{4}{|l|}{$\mathrm{HCV}$} \\
\hline AIC & $13,992.7$ & $13,949.1$ & $13,974.7$ \\
\hline c-index & 0.673 & 0.686 & 0.679 \\
\hline \multicolumn{4}{|l|}{ HBV } \\
\hline AIC & $1,886.4$ & $1,883.8$ & $1,917.4$ \\
\hline c-index & 0.750 & 0.758 & 0.726 \\
\hline \multicolumn{4}{|l|}{ NBNC } \\
\hline AIC & $3,341.4$ & $3,334.6$ & $3,359.5$ \\
\hline c-index & 0.682 & 0.689 & 0.679 \\
\hline
\end{tabular}

AIC, Akaike's information criterion; ALBI, albumin-bilirubin; mALBI, modified ALBI; PALBI, platelet-albumin-bilirubin; TNM, tumor node metastasis classification of the Liver Cancer Study Group of Japan, 6th edition; ALBI-T, ALBI-TNM score; mALBI-T, mALBI-TNM score; PALBI-T, PALBI-TNM score; HCV, hepatitis C virus; HBV, hepatitis B virus; NBNC, negative for both HBV and HCV.

\section{Disclosure Statement}

None of the authors has financial conflicts of interest to disclose concerning this study.

\section{References}

1 Hiraoka A, Kumada T, Kudo M, et al: Albumin-bilirubin (ALBI) grade as part of the evidence-based clinical practice guideline for HCC of the Japan Society of Hepatology: a comparison with the liver damage and ChildPugh classifications. Liver Cancer 2017;6:204-215.

2 Liu PH, Hsu CY, Hsia CY, et al: ALBI and PALBI grade predict survival for HCC across treatment modalities and BCLC stages in the MELD era. J Gastroenterol Hepatol 2017;32:879-886.

3 Hiraoka A, Kumada T, Michitaka K, et al: Usefulness of albumin-bilirubin grade for evaluation of prognosis of 2,584 Japanese patients with hepatocellular carcinoma. J Gastroenterol Hepatol 2016;31:1031-1036.

4 Hiraoka A, Michitaka K, Kumada T, et al: Validation and potential of albumin-bilirubin grade and prognostication in nationwide survey of 46,681 HCC patients in Japan - need for more detailed evaluation of hepatic function. Liver Cancer 2017;6:325-336. 